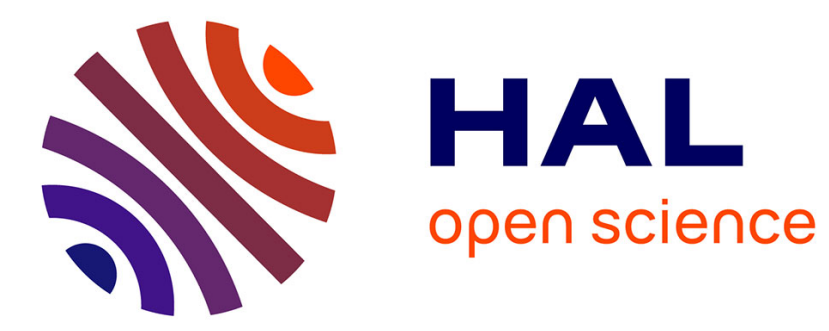

\title{
Dix arguments pour réviser le traité de Maastricht
}

André Grjebine

\section{To cite this version:}

André Grjebine. Dix arguments pour réviser le traité de Maastricht. Revue d'économie financière, 1992, 2 (Hors-série), pp.97-103. 10.3406/ecofi.1992.4604 . hal-01009719

\section{HAL Id: hal-01009719 \\ https://hal-sciencespo.archives-ouvertes.fr/hal-01009719}

Submitted on 18 Jun 2014

HAL is a multi-disciplinary open access archive for the deposit and dissemination of scientific research documents, whether they are published or not. The documents may come from teaching and research institutions in France or abroad, or from public or private research centers.
L'archive ouverte pluridisciplinaire $\mathbf{H A L}$, est destinée au dépôt et à la diffusion de documents scientifiques de niveau recherche, publiés ou non, émanant des établissements d'enseignement et de recherche français ou étrangers, des laboratoires publics ou privés.

\section{(1) $(1) \Theta$}

Distributed under a Creative Commons Attribution - NonCommercial - NoDerivatives| 4.0 


\title{
Persée
}

\author{
http://www.persee.fr
}

\section{Dix arguments pour réviser le traité de Maastricht}

\author{
André Grjebine \\ Grjebine André. Dix arguments pour réviser le traité de Maastricht. In: Revue d'économie financière. Hors-série, 1992. \\ Le Traité de Maastricht : quelles conséquences pour l'Europe financière ? . pp. 97-103.
}

Voir l'article en ligne

\section{Avertissement}

L'éditeur du site «PERSEE » - le Ministère de la jeunesse, de l'éducation nationale et de la recherche, Direction de l'enseignement supérieur, Sous-direction des bibliothèques et de la documentation - détient la propriété intellectuelle et les droits d'exploitation. A ce titre il est titulaire des droits d'auteur et du droit sui generis du producteur de bases de données sur ce site conformément à la loi n`98-536 du 1 er juillet 1998 relative aux bases de données.

Les oeuvres reproduites sur le site «PERSEE » sont protégées par les dispositions générales du Code de la propriété intellectuelle.

Droits et devoirs des utilisateurs

Pour un usage strictement privé, la simple reproduction du contenu de ce site est libre.

Pour un usage scientifique ou pédagogique, à des fins de recherches, d'enseignement ou de communication excluant toute exploitation commerciale, la reproduction et la communication au public du contenu de ce site sont autorisées, sous réserve que celles-ci servent d'illustration, ne soient pas substantielles et ne soient pas expressément limitées (plans ou photographies). La mention Le Ministère de la jeunesse, de l'éducation nationale et de la recherche, Direction de l'enseignement supérieur, Sous-direction des bibliothèques et de la documentation sur chaque reproduction tirée du site est obligatoire ainsi que le nom de la revue et- lorsqu'ils sont indiqués - le nom de l'auteur et la référence du document reproduit.

Toute autre reproduction ou communication au public, intégrale ou substantielle du contenu de ce site, par quelque procédé que ce soit, de l'éditeur original de l'oeuvre, de l'auteur et de ses ayants droit.

La reproduction et l'exploitation des photographies et des plans, y compris à des fins commerciales, doivent être autorisés par l'éditeur du site, Le Ministère de la jeunesse, de l'éducation nationale et de la recherche, Direction de l'enseignement supérieur, Sous-direction des bibliothèques et de la documentation (voir http://www.sup.adc.education.fr/bib/ ). La source et les crédits devront toujours être mentionnés. 


\title{
DIX ARGUMENTS POUR RÉVISER LE TRAITÉ DE MAASTRICHT
}

\author{
ANDRÉ GRJEBINE,
}

Fondation Nationale des Satences Poltiques,

Centre d'Etudes et de ReCherches Internationales

a création d'une monnaie unique s'inscrit dans la dynamique de l'intégration européenne ${ }^{1}$. Elle devrait constituer une étape majeure, d'une portée symbolique considérables, dans l'avancement vers un Etat fédéral européen ayant une puissance comparable ou supérieure à celle des Etats-Unis et du Japon. Mais, cette création ne doit pas se faire au détriment du développement économique et de l'équilibre social des pays membres.

Les structures économiques, sociales et culturelles des pays européens demeurent sensiblement différentes. Certes, les taux d'inflation se sont rapprochés ces dernières années et celui de la France est même inférieur actuellement à celui de l'Allemagne. Mais, c'est au prix de trois millions de chômeurs. En passant outre à ces différences, on risque de provoquer des situations explosives. Dans son dernier rapport annuel, publié en juin 1992, la Banque des Règlements Internationaux observe (pp. 143-144) que « si rien n'indique que tous les pays sont tenus de chercher à respecter les critères dès les premières évaluations, prévues pour 1996 et 1998, les efforts pour y parvenir pourraient rendre les taux de change et peut-être la politique budgétaire trop rigides, dans certains pays, pendant les années à venir ».

Le processus choisi pour parvenir à la monnaie unique est donc fondamental. Trois approches de la construction monétaire européenne étaient concevables. Selon la première, le passage d'une monnaie commune à la monnaie unique aurait été l'aboutissement d'un processus, sans doute long, de rapprochement des structures économiques et sociales des pays européens. Dans un premier temps, la priorié aurait donc été donnée à la mise en œuvre de mesures visant à favoriser ce rapprochement. La seconde

1 Pour une analyse critique plus détaillée du traité de Maastricht, le lecteur peut se reporter à mon étude publiée dans Le débat en septembre 1992: "Après Maastricht: des écus et des chômeurs?" Avec des commentaires critiques de Philippe Lagayette et Pierre Jacquet. 
approche aurait mis l'accent sur des critères de convergence structurels et pas seulement monétaires. Elle ouvrait la voie à une «union à plusieurs vitesses ». Cette approche a les faveurs de beaucoup d'économistes qui espèrent encore orienter dans ce sens l'application du traité de Maastricht. La dernière consistait à avancer vers la monnaie unique en passant outre aux différences culturelles, sociales et économiques fondamentales qui subsistent entre les pays membres, y compris entre la France et l'Allemagne. C'est celle qui a prévalu à Maastricht.

L'objet de cet article est de mettre en évidence les risques inhérents à cette stratégie. Il serait évidemment absurde d'assimiler cette critique de la construction monétaire européenne à des manifestations de nationalisme anti-européen. La contestation des aspects monétaires des Accords de Maastricht n'implique aucune hostilité à l'égard de la construction européenne mais résulte simplement du souci de ne pas sacrifier pour autant les valeurs qui ont longtemps distinguées l'Europe occidentale des autres parties du monde: la conjonction de l'efficacité économique et de la solidarité sociale.

1) L'intégration des marchés du travail sera infiniment plus lente que celle des marchés de capitaux, de biens et de services. Même aux Etats-Unis, l'unité monétaire ne suffit pas à assurer l'homogénéisation des taux de croissance. Certains Etats connaissent de longue période de forte croissance, d'autres ont durablement des taux de croissance très inférieurs à la moyenne nationale. En particulier, l'évolution de l'emploi est très différente d'un Etat à l'autre, notamment parce que l'intégration renforce la division du travail et favorise ainsi des spécialisations étroites des Etats, par industrie et par produit. Ceux-ci sont alors affectés par des fluctuations spécifiques en fonction des modifications de la demande de biens dans la production desquels ils se sont spécialisés. La flexibilité des salaires, tant vantée aux Etats-Unis, s'avère insuffisante pour rétablir la compétitivité et préserver l'emploi. Seule la mobilité de la main-d'œuvre permet de réduire les écarts régionaux de taux de chômage. Or, compte tenu de la diversité des langues et des traditions culturelles et sociales qui caractérise l'Europe, la mobilité entre pays européens sera forcément beaucoup plus faible qu'elle ne l'est aux Etats-Unis. Du reste, en supposant même que la mobilité inter-étatique s'accentue, qui accepterait que l'intégration européenne se solde par le déclin de certains pays et une constante montée en puissance d'autres?

2) même si la croissance démographique s'est ralentie dans l'ensemble des pays européens, tous ne sont pas également atteints. De ce fait, pour stabiliser le chômage, la croissance doit être sensiblement plus rapide en France ou en Espagne que dans un pays à démographie stagnante comme l'Allemagne.

3) Les principales relations macroéconomiques, les structures économi- 
ques et sociales comme les modalités d'insertion dans la division internationale du travail sont encore sensiblement différentes d'un pays européen à l'autre. Les effets des politiques éconmiques sont, de ce fait, également assez différents. Des règles communautaires uniformes ne pourraient donc que renforcer les disparités entre les pays européens. Contrairement à une idée reçues, une véritable convergence des pays européens exigerait des politiques foncièrement différentes d'un pays à l'autre, de manière à permetre aux plus faibles de rattraper les mieux armés. La similitude des politiques économiques ne pourrait, dans le meilleur des cas, que renforcer le statu quo.

4) Compte tenu de ces différences, les fluctuations d'une monnaie unique ou de monnaies durablement, sinon définitivement liées entre elles par un taux de change fixe - peuvent aller à l'encontre des intérêts particuliers de certains pays. De surcroît, on ne peut exclure que des perturbations locales appellent des réponses spécifiques, par exemple la modification du taux de change global. Il n'y a a priori aucune raison de supposer que le taux de change commun sera optimal pour tous les pays membres.

5) L'unification des marchés de capitaux ne signifie pas que tous les Etats seront traités de la même manière. De même qu'une PME et une grande entreprise ayant une réputation solidement établie doivent emprunter au sein d'un même pays à des taux sensiblement différents, on voit mal pourquoi il n'en serait pas de même pour deux Etats ayant des politiques nettement différentes. De fait, aux Etats-Unis, une prime de risque non négligeable est appliquée aux Etats surendettés et ceux dont la politiques budgétaires sont jugées imprudentes. Avec des taux d'intérêt réels élevés et de faibles taux de croissance, et sans possiblité d'un financement monétaire du déficit budgétaire, le paiement des intérêts va engendrer des déficits budgétaires futurs croissants.

6) La Communauté n'a pas les moyens de se substituer aux Etats pour mener une politique anticyclique. En effet, elle ne dispose pas des ressources suffisantes pour mener une politique conjoncturelle, le budget communautaire ne représentant qu'une infime fraction de celui des Etats membres. Aux Etats-Unis, le budget fédéral est plus élevé que la somme des Etats et collectivités locales. L'augmentation des transferts en provenance de l'Etat fédéral compenserait de 30 à $40 \%$ de la variation de revenu résultant d'un choc régional. L'absence d'un effet anti-cyclique propre au budget de la Communauté ne tient pas seulement à sa taille relativement réduite comparée aux budgets nationaux, mais aussi au fait qu'il est principalement alimenté par des contributions des Etats et non par des recettes fiscales directes qui augmentent ou diminuent automatiquement en fonction de la conjoncture. Dans la logique de Maastricht, les Etats européens devraient rapidement trasférer au niveau communautaire une part notable de leurs 
recettes et de leurs dépenses publiques. Malgré les efforts acharnés déployés par la commission pour accroître rapidement le budget communautaire, le moins qu'on puisse dire est que cette perspective parait encore lointaine. En renonçant à des politiques anticycliques nationales, les européens renonceront donc à toute politique de régulation de la conjoncture au sein de l'espace européen.

7) Les espoirs placés dans une politique régionale européenne paraissent illusoires. L'échec des politiques régionales menées en France ou au Roayme-Uni augure mal des chances de succès d'une telle politique à l'échelle européenne. Il parait douteux que les pays riches de la Communauté consentent un effort à la taille des problèmes qui se posent.

8) La puissance d'une économie dépend, d'abord, de ses performances en matière de formation, de recherche-développement, de consensus social. Celles-ci donnent aux économies les plus fortes la marge de manœuvre pour consolider leur avance. On pourrait penser que l'exemple de l'Allemagne va inspirer les autres pays européens. En réalité, la concurrence à laquelle ils doivent faire face et la priorité accordée à la stabilité monétaire leur imposent de réduire au maximum leurs dépenses publiques et privées non immédiatement rentables, c'est-à-dire précisément celles qui préparent le long terme. La nécessité de se conformer aux indicateurs de convergence va également conduire les pays les plus faibles à réduire leurs dépenses d'investissement, plus faciles à comprimer que les dépenses de fonctionnement. Dans certains de ces pays, notamment en France, l'obsession du court terme est exacerbée par le développement des OPA et l'instabilité de l'épargne institutionnelle, principalement placée en SICAV et fonds communs de placement, qui obligent les dirigeants à rendre des comptes à leurs actionnaires quasiment au jour le jour. Enfin, par crainte d'une fuite des capitaux, les autorités monétaires maintiennent des taux d'intérêt élevés qui détournent une large partie de l'épargne vers les placements quasi liquides ou à court terme. Dans ce contexte, l'écart entre les économies européennes ne pourrait que s'élargir. A moins que les mutations politiques, économiques et sociales en cours dans certains pays européens ne viennent modifier les comportements des agents économiques dans ces pays.

9) Rappelant l'exemple de l'unification allemande, la Banque des règlements Internationaux (rapport cité, p. 145) exprime la crainte qu'à mesure que l'intégration des marchés du travail progessera en Europe, les pays les moins développés de la Communauté ne revendiquent des salaires équivalents à ceux des pays riches au mépris des écarts de productivité, ce qui aurait « des conséquences indésirables sur le chômage ». A première vue, des performances similaires en matière de productivité - et donc dans une large mesure de compétivité dans un système de taux de change immuablement fixes - peuvent être obtenues sans que tous les coûts soient 
identiques d'uns pays à l'autre. Seule la composante totale doit l'être, même si la répartition est différente. Il n'en demeure pas moins que la compensation d'un coût supérieur par des coûts plus faible sera d'autant plus limitée qu'au fur et à mesure du renforcement de la construction européenne, les solidarités catégorielles vont se développer, des effets du mimétisme vont se produire, favorisant la propagation des avantages acquis dans un pays chez ses partenaires. Il sera de plus en plus difficile d'expliquer aux salariés qu'ils doivent accepter des rémunération plus faibles que leurs collègues d'autres régions sous prétexte que les entreprises qui les emploient sont mal gérées, les équipements plus anciens ou les charges sociales plus lourdes. Dans cette optique, on peut s'attendre que les syndicats français revendiquent tôt ou tard des salaires ou des conditions de travail (la semaine de 35 heures par exemple) similaires à ceux qu'obtiendront leurs partenairs les plus avancés, notamment les syndicats allemands. Une course de vitesse va ainsi s'engager entre ce processus spontané de contagion et l'action volontaire des dirigeants d'entreprise et des gouvernements pour rénover les structures économiques et sociales des entreprises et les mettre à niveau avec les plus performantes de la Communauté. Ce n'est que dans l'hypothôse peu probable - où cette restructuration sera la plus rapide que la construction europénne aura un impact convergent sur les économies des pavs membres.

10) Certes, la suppression des monnaies nationales éliminera le risque de spéculation contre la monnaie du pays qui adoptera une politique «expansionniste». Le passage à une monnaie unique ne supprimera pas pour autant la contrainte extérieure qui amène un pays, dans l'état actuel des choses, à freiner sa croissance pour réduire ses importations et éviter ainsi un solde commercial négatif avec ses partenaires. L'entrée en vigueur de l'Acte unique le $1^{\text {er }}$ janvier 1993 entrainera la suppression des contrôles douaniers entre pays de la Communauté et, par voie de conséquence, la disparition des statistiques douanières traditionnelles. Mais l'INSEE comme les instituts similaires des autres pays européens sont en train de préparer de nouvelles modalités de perception des flux commerciaux entre pays europens, les enquêtes auprès des entreprises devant se substituer aux statistiques douanières. La création d'une monnaie unique n'entrainera pas, non plus, la disparition des balances de paiements entre pays européens. Il est vrai qu'on ne comptabilise pas les flux entre, par exemple, la Bourgogne, la Bretagne et l'Ile-de-France. C'est qu'il n'existe pas, pour des raisons historiques, de « comptabilité nationale » au niveau des régions. Pour qu'il en soit de même à l'échelle europénne, il faudrait substituer une comptabilité strictement européenne aux comptes nationaux, faute de quoi le nécessité d'ajuster ces derniers fera apparaître des soldes positifs ou négatifs, comme c'est le cas actuellement. La fermeture de l'INSEE et de ses équivalents dans les autres 
pays européens au profit d'un Institut européen de la statistique n'est pas à l'ordre du jour.

\section{Peut-on "aménager » le traité de Maastricht?}

De nombreux économistes conscients des difficultés que risque de susciter la mise en œuvre du traité de Maastricht craignent néanmoins de manquer de réalisme en sortant du cadre défini par la libération des mouvements de capitaux, le SME et ce traité. Ils expliquent que le traité de Maastricht doit être ratifié, malgré ses imperfections, quitte ensuite à l'appliquer d'une manière moins conforme à l'orthodoxie qui a présidé à son élaboration.

Il n'est jamais sain de ratifier un traité en espérant que son application permettra de dégager une marge de manœuvre qu'il ne prévoit pas. Il est à craindre qu'il ne s'agisse, de surcroît, d'un faux espoir. L'idée d'un aménagement du traité de Maastricht se heurte à la surveillance que vont exercer sur leurs partenaires, les autorités allemandes, mais aussi, à une sorte de surenchère où chaque gouvernement entend surpasser les autres dans la voie de l'orthodoxie et éprouve une extrême satisfaction à leur donner des leçons de conformisme. Quand on voit la suspicion avec laquelle les autorités monétaires de l'Allemagne, voire l'opinion publique de ce pays, observent la mise en place d'une union économique et monétaire, allant même jusqu'à s'interroger sur les modalités utilisées dans les autres pays pour calculer le déficit budgétaire, on ne peut qu'être sceptique sur la marge de manœuvre qui sera impartie à un pays membre de l'UEM. On peut compter sur la vigilance des autorités allemandes pour ramener dans le droit chemin le pays qui tenterait d'échapper aux obligations auxquelles il aura souscrit.

Dans ce contexte, on est amené à considérer que la monnaie unique devrait être le résultat d'un processus de rapprochement des économies européennes. Les promoteurs du traité de Maastricht expliquent que la voie tracée ne peut plus être remise en cause pour des raisons politiques. Ce faisant, ils ne font qu'ancrer encore plus dans les esprits l'opinion selon laquelle il n'y pas de solution alternative. Ils risquent ainsi de préciter le mal qu'ils voient partout aujourd'hui : s'il n'y a pas d'autre option pour la construction europénne que celle que prévoit le traité de Maastricht, il est à craindre que le chômage continuant à progresser, le rejet de l'idée européenne ne prenne de plus en plus d'ampleur. La réalité des faits pourrait bien un jour s'imposer au détriment d'une approche de la construction européenne qui prétend passer outre aux différences structurelles qui subsistent encore entre les principaux pays membres.

Pour éviter que cette remise en question ne soit par trop brutale, les grouvernements des pays signataires seraient bien inspirés d'élargir leur conception de la convergence. Par exemple, plutôt que de privilégier, en tant 
que critère de convergence, le seul taux d'inflation comme le fait le traité de Maastricht, il aurait sans doute été plus judicieux d'adopter un indicateur mesurant le taux d'inflation pour un même taux de chômage. Il est clair qu'obtenir $3 \%$ d'inflation avec $10 \%$ de chômage n'a pas le même signification que d'avoir le même taux d'inflation avec un chômage beaucoup plus faible. Il serait donc logique de ramener le taux d'inflation mesuré au taux qui serait obtenu avec un taux de chômage jugé acceptable, par exemple $5 \%$. En pratique, un tel calcul serait évidemment ardu et sujet à contestation, on pourait donc le remplacer par une formule moins satisfaisante d'un point de vue scientiques, mais plus facile à circuler en remplaçant l'indicateur "taux d'inflation» par un "taux de malaise » résultant de l'addition du taux d'inflation et du taux de chômage.

\section{Tableau 1}

Principaux taux de chômage + taux d'inflation

\begin{tabular}{rrrrrrrr}
\hline \hline & France & All. Ouest & Roy. Uni & Italie & Espagne & Etats-Unis & Suède \\
\hline TCE $^{1}$ & $+1,7$ & $+18,7$ & $-13,2$ & $+2,0$ & $+10,2$ & $-35,2$ & $-7,0$ \\
\hline 1981 & 20,4 & 10,6 & 21,0 & 25,9 & 28,2 & 16,7 & 14,9 \\
1982 & 19,7 & 11,2 & 20,0 & 25,3 & 30,1 & 15,2 & 13,8 \\
1983 & 18,0 & 11,0 & 17,2 & 24,0 & 29,3 & 13,6 & 14,7 \\
1984 & 17,4 & 9,9 & 16,7 & 21,2 & 30,6 & 11,2 & 10,8 \\
1985 & 15,9 & 9,2 & 16,6 & 18,6 & 29,3 & 10,4 & 9,8 \\
1986 & 13,1 & 5,9 & 15,6 & 16,3 & 29,4 & 9,3 & 7,3 \\
1987 & 13,7 & 6,8 & 14,6 & 15,9 & 25,8 & 10,7 & 7,2 \\
1988 & 12,7 & 17,6 & 13,5 & 16,3 & 24,2 & 9,3 & 7,6 \\
1989 & 12,8 & 8,7 & 12,6 & 17,1 & 23,5 & 9,7 & 8,6 \\
1990 & 11,8 & 7,7 & 12,9 & 16,2 & 22,3 & 10,4 & 10,8 \\
1991 & 12,6 & 7,8 & 14,8 & 16,3 & 21,9 & 10,8 & 12,0 \\
& & & & & & & \\
\hline \hline
\end{tabular}

1. Evolution du taux de change effectif entre les Accords du Plaza (16/9/1985) et le 5/11/1991. Source: Perspectives économiques de l'OCDE, décembre 1991. 